\title{
PLANTS VISITED BY Apis mellifera L. (HYMENOPTERA: APIDAE) IN RECÔNCAVO BAIANO, STATE OF BAHIA, BRAZIL
}

\author{
Andreia Santos do Nascimento ${ }^{1}$, Carlos Alfredo Lopes de Carvalho ${ }^{1}$, Márcio Lacerda Lopes \\ Martins ${ }^{1}$ \\ ${ }^{1}$ Universidade Federal do Recôncavo da Bahia - E-mails: asndea@yahoo.com.br, calfredo@ufrb.edu.br, marciollm@ufrb.edu.br
}

\section{ABSTRACT}

The objective of this study was to identify the plants visited by Apis mellifera L. (Hymenoptera: Apidae) in the region of Recôncavo Baiano, State of Bahia, Brazil. Flowering plants were assessed in a radius of $1.500 \mathrm{~m}$ around the apiaries visited. A total of 240 species belonging to 171 genera and 50 families were recorded. The family Leguminosae had the highest species richness $(22 \%)$, followed by Asteraceae (14\%) and Malvaceae (7\%) of all plant species visited by Apis mellifera. The highest percentage of plants collected had herbaceous growth habit (44\%), followed by woody plants (26\%) and shrubs (18\%). Lianas, vines and palm trees were significantly less represented. It was found that $65 \%$ of species had actinomorphic flowers and $33 \%$ had zygomorphic flowers. The genera that showed greater diversity were Sida with nine species, Croton with six species, Senna with five species. and while Chamaecrista, Eucalyptus, Eupatorium, Ocimum, Passiflora and Vernonia were represented by four species each. The flora of apicultural interest in the region of Recôncavo Baiano has a wide range of species with varying growth habits.

Keywords: Apicultural flora, bee, pollen, honey

\section{PLANTAS VISITADAS POR Apis mellifera L. (HYMENOPTERA: APIDAE) NO RECÔNCAVO BAIANO}

\section{RESUMO}

O objetivo deste estudo foi identificar as plantas visitadas por Apis mellifera L. na região do Recôncavo Baiano. Foi realizado um levantamento das plantas em floração em um raio de 1.500 m dos apiários visitados. Foram registradas 240 espécies, pertencentes a 171 gêneros e 50 famílias. A família Leguminosae apresentou maior riqueza de espécies com 22\%, seguida da Asteraceae (14\%) e Malvaceae (7\%) do total de espécies vistadas por Apis mellifera. A maior percentagem de 
plantas coletadas apresentou hábito de crescimento herbáceo com 44\%, seguido pelas de hábito arbóreo (26\%) e arbustivo (18\%). As lianas, trepadeiras e palmeiras tiveram menor representatividade. Verificou-se que 65\% das espécies apresentaram flores actinomorfas e 33\% zigomorfas. Os gêneros que apresentaram maior diversidade foram Sida com nove espécies, Croton com seis, Senna com cinco e Chamaecrista, Eucalyptus, Eupatorium, Ocimum, Passiflora e Vernonia representados por quatro espécies cada. A flora de interesse apícola na região do Recôncavo Baiano apresenta uma grande diversidade de espécies com hábitos de crescimento variado.

Palavras-chave: Flora apícola, abelha, pólen, mel

\section{INTRODUCTION}

Insects are important pollinators of angiosperms, and among them, the bees are considered the most efficient ones in almost all ecosystems. Approximately $67 \%$ of flowering plants depend on bees for pollination, while these depend directly on collecting pollen and nectar to feed their larvae and adults (VIANA \& SILVA, 2006).

Africanized bees feed almost exclusively on pollen and nectar, needing to visit certain amounts of flowers daily. They can better adapt to tropical climates, managing to survive for several months, even when they have to face long periods of drought or rain, very common in these regions (MUNIZ \& BRITO, 2007). The environmental value of apiculture is characterized by interdependence with the vegetation, since the visits of bees to flowers 98 of native species ensure agricultural pollination, increasing agricultural productivity and ensuring the perpetuation of native species (NOGUEIRA-NETO, 1998).

Melitophilous plants are those that provide products for bees (TORQUATO, 2006). According to Wiese (1987) the apicultural value of plants is related to the abundance of flowers, the daily volume of nectar secreted, with the concentration of sugars in nectar and low competition for pollinators.

The diversity of the Brazilian flora associated with the territorial extension and the existing climate variability, allows the country a great apicultural potential, with harvests during the entire year, what distinguishes Brazil from other producing countries that normally gather honey only once a year (MARCHINI et al., 2004). Little 
is known about the floral resources needed to maintain communities of bees in natural habitats in Brazil (AGUIAR, 2003) and knowledge of plants that are food resources for bees is essential to the establishment of conservation programs for these insects (CARVALHO \& MARCHINI, 1999). Through knowledge of plants used by bees in the pasture, beekeepers can handle their apiary in order to get a better use of flowers and thereby optimize their honey production (JONES \& BRYANT JR., 1996).

The apicultural activity in Recôncavo Baiano is developed mainly by small and medium-sized farms, becoming an important source of income for many families in rural areas. Despite its importance, there are some limitations that hinder the increase of production honey and other bee products in this region, among which the low information are the regional apicultural flora. Thus, the objective of this study was to identify the plants visited by Apis mellifera L. (Hymenoptera: Apidae) in the Recôncavo Baiano region.

\section{MATERIAL AND METHODS}

Monthly collections of vegetative and reproductive parts of flowering plants visited by bees within $1.500 \mathrm{~m}$ of the apiaries were conduct in the following six municipalities of Recôncavo Baiano: Cachoeira, Castro Alves, Cruz das Almas, Governador Mangabeira, Itatim and São Felix, in the period of March/2009 to February/2010.

The collection and processing of botanical material followed the techniques described by Moreti et al. (1989) and the material collected was deposited in the Herbarium of Recôncavo Baiano (HERB), Federal University of Recôncavo da Bahia. The pollen grains removed from the poliferous material (flower buds) were mounted on slides using the acetolysis method (ERDTMAN, 1960). The taxonomic identification of plant species was based on the available scientific literature and comparison with material deposited in the Herb collection.

The pollen slides were deposited in the pollen reference collection of Apiculture/Meliponiculture plants of the Núcleo de Estudo dos Insetos do Centro de Ciências Agrárias, Ambientais e Biológicas (Experimental Center for the Studies of Insects - Center of Agricultural, Environmental and Biological Sciences)/ Federal University of Recôncavo da Bahia. The characteristics of growth habit and floral 
symmetry for each species were recorded according to Ferri (1983) and Bell (1991).

\section{RESULTS AND DISCUSSION}

A total of 240 plant species were visited by Apis mellifera in the municipalities of Recôncavo Baiano, distributed in 171 genera and 50 families (Table 1). The Leguminosae family had a higher richness of species (52) representing $22 \%$ of the total, followed by Asteraceae (14\%) and Malvaceae (7\%) (Figure 1).

The Asteraceae family stands out in many studies (POTT \& POTT,1986; RAMALHO et al., 1990; MARCHINI et al., 2001 and MELO, 2008) as one of the richest in species visited by bees. Locatelli \& Machado (2001) suggest that this is probably due to the fact that this family is the largest and of greater geographical distribution one among angiosperms.

Melo (2008) recorded a total of 161 species of plants visited by $A$. mellifera, belonging to 117 genera and 46 families in the region of Mundo Novo, Bahia, and that the families Asteraceae, Leguminosae, Malvaceae, Myrtaceae, Sterculiaceae and Verbenaceae were the richest in species. These families were also more representative in this study (Figure 1).
The Leguminosae family, the most abundant in number of species in the studied region was reported by Viana et al. (2006) as one of the most important in terms of number of apicultural flora in an area of restinga in Bahia, with seven species recorded, indicating a likely apicultural potential for this region. The Leguminosae-Mimosoideae are composed of about 60 genera represented by 3,000 species, distributed mainly in tropical and subtropical regions, plus some species that are found in temperate regions. Its importance as a supplier of food source for bees is well known and is mentioned in several studies (ALMEIDA, 2002; ANDENA et al., 2002; CARVALHO et al., 2006 and NASCIMENTO et al., 2009a).

According to Ramalho et al. (1990) and Carvalho et al. (2001) the families Anacardiaceae, Arecaceae, Asteraceae, Balsaminaceae, Euphorbiaceae, Labiatae, Moraceae, Myrtaceae, Proteaceae, Rubiaceae and Sterculiaceae are important sources for the collection of trophic resources in the neotropical regions both for Africanized bees and stingless bees. 
Table 1. List of species of apicultural interest collected at flowering within $1.500 \mathrm{~m}$ of apiaries in Recôncavo Baiano: March/2009 to February/2010. Legend: GH $=$ Growth Habit, Herb = herbaceous, Arb = arboreous, Shr = shrubby, Li = Liana, Vine = vine, Pal = palm, FS = Flower Symmetry, Z = zygomorphic, A $=$ actinomorphic, $\mathrm{NR}=$ not registered, $\mathrm{RE}=$ Resource, $\mathrm{N}=$ nectar, $\mathrm{P}=$ pollen, $\mathrm{CA}=$ Cruz das Almas $, \mathrm{CC}=\mathrm{Cachoeira}, \mathrm{CT}=\mathrm{Castro} \mathrm{Alves}, \mathrm{GM}=\mathrm{Governador}$ Mangabeira, IT = Itatim, SAF = São Felix.

\begin{tabular}{|c|c|c|c|c|c|c|c|}
\hline Famíly & Scientific Name & Common Name & GH & FS & RAE & Place of collection & Flowering Period \\
\hline \multirow[t]{2}{*}{ Acanthaceae } & Justicia sp. & - & Shr & $\mathrm{Z}$ & $\mathrm{P}$ & $\mathrm{CA}$ & May to August \\
\hline & Ruellia paniculata $\mathrm{L}$. & - & Herb & $\mathrm{Z}$ & $\mathrm{N} / \mathrm{P}$ & $\mathrm{CT}$ & Jan. and Sep. \\
\hline \multirow{3}{*}{ Amaranthaceae } & Alternanthera sp. & - & Shr & $\mathrm{Z}$ & $\mathrm{N}$ & $\mathrm{CA}$ & April; July to Aug. \\
\hline & Alternanthera tenella Colla & Perpétua do mato/ apaga-fogo & Herb & $\mathrm{Z}$ & $\mathrm{N}$ & $\mathrm{CT}$ & Aug. to Oct. \\
\hline & Amaranthus spinosus L. & Thorny amaranth & Herb & $\mathrm{Z}$ & $\mathrm{P}$ & $\mathrm{CA}$ & Aug. \\
\hline \multirow{6}{*}{ Anacardiaceae } & Astronium cf. macrocalyx Engl. & - & Arb & A & - & CT & Oct. to Dec. \\
\hline & Astronium sp. & Brazilian pepper & Arb & $\mathrm{A}$ & - & $\mathrm{CA}$ & March to May; Oct. to Dec. \\
\hline & Mangifera indica $\mathrm{L}$. & Mango & Arb & A & $\mathrm{N}$ & $\mathrm{CC}, \mathrm{CA}, \mathrm{CT}, \mathrm{GM}$ & Sep. \\
\hline & Schinus terebinthifolius Raddi & Brazilian pepper tree & Shr & $\mathrm{A}$ & $\mathrm{N}$ & $\mathrm{CA}, \mathrm{CC}, \mathrm{GM}$ & Feb. to May \\
\hline & Spondias tuberosa Arr. Cam. & Umbu/Brazil plum & Arb & A & - & $\mathrm{CA}$ & Oct. \\
\hline & Spondias sp. & Cajarana of the Hinterland & Arb & A & $\mathrm{N}$ & $\mathrm{CA}$ & Dec. \\
\hline Apiaceae & Coriandrum sativum $\mathrm{L}$. & Coriander & Herb & $\mathrm{A}$ & - & $\mathrm{CA}$ & Sep. to Dec. \\
\hline \multirow[t]{2}{*}{ Apocynaceae } & Tabernaemontana laeta Mart. & Jasmine & Arb & A & - & $\mathrm{CC}$ & Sep. \\
\hline & Thevetia thevetioides (Kunth) K. Schum & Giant helvetia & Shr & A & - & $\mathrm{CA}$ & Nov. \\
\hline \multirow[t]{3}{*}{ Arecaceae } & Cocos nucifera $\mathrm{L}$. & Coconut & Pal & A & $\mathrm{N}$ & $\mathrm{CA}$ & Oct. to Jan.; April to July \\
\hline & Elaeis guineensis Jacq. & Oil palm & Pal & A & $\mathrm{P}$ & $\mathrm{CA}$ & March to April; Sep. to Aug. \\
\hline & Syagrus coronata Bond. & Licuri palm & Pal & A & $\mathrm{N}$ & $\mathrm{CA}$ & Oct. to Dec. \\
\hline \multirow[t]{6}{*}{ Asteraceae } & Acanthospermum australe (Loefl.) Kuntze. & Paraguay Starbur & Herb & A & - & $\mathrm{CA}$ & Aug. to Sep. \\
\hline & Acanthospermum sp. & Bristly Starbur & Herb & A & - & $\mathrm{CA}$ & Aug. to April. \\
\hline & Ageratum conyzoides $\mathrm{L}$. & Invading Ageratum & Herb & $\mathrm{A}$ & $\mathrm{N}$ & CA, SAF & Jan. to Dec. \\
\hline & Ageratum sp. & Whiteweed & Herb & $\mathrm{A}$ & - & $\mathrm{CC}$ & Oct. \\
\hline & Aspilia sp. & Daisy; Emilia plant & Herb & A & - & $\mathrm{CA}$ & July \\
\hline & $\begin{array}{l}\text { Bidens pilosa } \mathrm{L} . \\
\text { Bidens sulphurea (Cav.) Sch. Bip. }\end{array}$ & $\begin{array}{l}\text { Hairy beggarticks } \\
\text { Sulfur Cosmos; Yellow cosmos }\end{array}$ & $\begin{array}{l}\text { Herb } \\
\text { Herb }\end{array}$ & $\begin{array}{l}\mathrm{A} \\
\mathrm{A}\end{array}$ & $\begin{array}{l}\mathrm{N} / \mathrm{P} \\
\mathrm{N} / \mathrm{P}\end{array}$ & $\begin{array}{c}\mathrm{CC}, \mathrm{CA}, \mathrm{CT}, \mathrm{GM} \\
\mathrm{CA}\end{array}$ & $\begin{array}{l}\text { May. to June } \\
\text { Jan. to Aug. }\end{array}$ \\
\hline
\end{tabular}


PLANTS VISITED BY Apis mellifera L. (HYMENOPTERA: APIDAE) IN RECÔNCAVO BAIANO, STATE OF BAHIA, BRAZIL

Table 1. Continue...List of species of apicultural interest collected at flowering within $1.500 \mathrm{~m}$ of apiaries in Recôncavo Baiano: March/2009 to February/2010.

\begin{tabular}{|c|c|c|c|c|c|c|c|}
\hline Famíly & Scientific Name & Common Name & HC & SF & RE & Place of collection & Flowering Period \\
\hline \multirow{22}{*}{ Asteraceae } & Bidens sp. & - & Herb & A & $\mathrm{N}$ & $\mathrm{CA}$ & Feb. to April; July to Sep. \\
\hline & Blainvillea biaristata DC. & - & Herb & A & - & $\mathrm{CA}$ & Aug. \\
\hline & CentratHerbum punctatum Cass. & Brazilian button flower & Herb & A & $\mathrm{N}$ & $\mathrm{CC}, \mathrm{CA}, \mathrm{CT}, \mathrm{GM}$ & May to Aug. \\
\hline & Conyza canadensis (L.) Cronquist. & Canadian horseweed & Herb & A & - & $\mathrm{CA}$ & March to June \\
\hline & Eclipta alba (L.) Hassk. & Elephant's foot & Herb & A & - & $\mathrm{CA}$ & Aug. \\
\hline & Erechtites hieraciifolius (L.) Raf. Ex DC. & Fireweed & NR & A & - & $\mathrm{CA}$ & Feb. to April; Sep. to Nov. \\
\hline & Erechtites sp. & Redflower ragleaf & Herb & A & $\mathrm{N}$ & $\mathrm{CA}$ & July \\
\hline & Eupatorium maximilianii Schrad. & - & Herb & A & - & $\mathrm{CA}$ & Aug. \\
\hline & Eupatorium sp.1 & - & Herb & A & - & $\mathrm{CA}$ & Aug. \\
\hline & Eupatorium sp.2 & False nettle & Herb & A & - & $\mathrm{CC}$ & Sep. \\
\hline & Eupatorium sp. 3 & Little Joe & Herb & A & & $\mathrm{CC}$ & Oct. \\
\hline & Galinsoga parviflora Cav. & Small-flower galinsoga & Herb & A & $\mathrm{P}$ & $\mathrm{CA}$ & May to July \\
\hline & Melampodium paniculatum Gardner & Butter daisy & Herb & A & - & $\mathrm{CA}$ & Aug. to Sep. \\
\hline & Melampodium sp.1 & - & Herb & A & - & $\mathrm{CA}$ & Aug. \\
\hline & Melampodium sp.2 & - & Herb & A & - & $\mathrm{CC}$ & Sep. \\
\hline & Montanoa pyramidata Sch.Bip. & Margarida-branca & $\mathrm{Shr}$ & A & - & $\mathrm{CA}$ & July to Oct. \\
\hline & Piptocarpha sp. & Ash daisy & Arb & A & - & $\mathrm{CT}$ & Dec. \\
\hline & Pluchea sagittalis (Lam.) & - & Herb & A & - & $\mathrm{CA}$ & Oct. \\
\hline & Rudbeckia laciniata $\mathrm{L}$. & Cutleaf coneflower & Herb & A & - & $\mathrm{CA}$ & Oct. to Jan. \\
\hline & Sonchus oleraceus L. & - & Herb & A & - & $\mathrm{CA}$ & July \\
\hline & Vernonia condensata Baker. & Boldus & Shr & A & $\mathrm{N}$ & $\mathrm{CA}$ & June to Nov. \\
\hline & Vernonia sp.1 & Bitterleaf & Shr & A & $\mathrm{N}$ & $\mathrm{CA}$ & Oct.to March \\
\hline \multirow{4}{*}{ Bignoniaceae } & Vernonia sp.2 & Bitterleaf & Shr & $\mathrm{A}$ & $\mathrm{N}$ & $\mathrm{CA}$ & Oct. to March \\
\hline & Vernonia sp.3 & - & Herb & $\mathrm{A}$ & $\mathrm{N}$ & $\mathrm{CA}$ & Aug. \\
\hline & Jacaranda sp. & Brazilian rose wood & Arb & $\mathrm{Z}$ & - & $\mathrm{CC}$ & Oct. \\
\hline & $\begin{array}{l}\text { Spathodea campanulata P. Beauv. } \\
\text { Stenolobium stans } \text { (L.) Seem. }\end{array}$ & $\begin{array}{l}\text { African Tuliptree } \\
\text { Yellow trumpetbush }\end{array}$ & $\begin{array}{l}\text { Arb } \\
\text { Arb }\end{array}$ & $\begin{array}{l}\mathrm{Z} \\
\mathrm{Z}\end{array}$ & $\begin{array}{l}P \\
-\end{array}$ & $\begin{array}{l}\text { CA, CC } \\
\text { CA }\end{array}$ & $\begin{array}{l}\text { Aug. to Nov. } \\
\text { Jan. to Dec. }\end{array}$ \\
\hline
\end{tabular}


Table 1. Continue...List of species of apicultural interest collected at flowering within 1.500 m of apiaries in Recôncavo Baiano: March/2009 to February/2010.

\begin{tabular}{|c|c|c|c|c|c|c|c|}
\hline Family & Scientific Name & Common Name & HC & FS & $\mathbf{R E}$ & Place of collection & Flowering Period \\
\hline & Tabebuia sp. & Brazilian walnut & Arb & $\mathrm{Z}$ & $\mathrm{P}$ & $\mathrm{CA}$ & May to Oct. \\
\hline Bignoniaceae & Tecoma heptaphylla (Vell.) Mart. & Pink tecoma & Arb & $\mathrm{Z}$ & $\mathrm{N}$ & IT & May to July \\
\hline Bombacaceae & Pachira aquatica Aubl. & Guaianan chestnut & Shr & A & - & $\mathrm{CA}$ & Dec. \\
\hline \multirow[t]{3}{*}{ Boraginaceae } & Cordia superba Cham. & Baba-de-boi & Shr & A & - & $\mathrm{CA} ; \mathrm{CT}$ & Jan. \\
\hline & Cordia verbenacea DC. & Forget-me-not & Shr & A & - & $\mathrm{CA}$ & Sep. \\
\hline & Heliotropium sp. & Heliotrope & Herb & A & $\mathrm{N}$ & $\mathrm{CC}, \mathrm{CA}$ & Aug. \\
\hline Capparaceae & Crataeva tapia $\mathrm{L}$. & Tapia & Arb & $\mathrm{Z}$ & - & $\mathrm{CT}$ & Jan.; Nov. \\
\hline Caprifoliaceae & Sambucus australis Cham \& Schlecht. & Black elder & Shr & A & $\mathrm{P}$ & $\mathrm{CA}$ & Sep. to April \\
\hline Chrysobalanaceae & Licania tomentosa Benth. & Oiti tree & Arb & $\mathrm{Z}$ & - & CA & Oct. to Nov. \\
\hline Combretaceae & Terminalia catappa $\mathrm{L}$. & Bengal Almond & Arb & A & - & $\mathrm{CA}$ & Sep. \\
\hline Commelinaceae & Commelina benghalensis L. & Bengal dayflower & Herb & $\mathrm{Z}$ & - & $\mathrm{CA} ; \mathrm{CT}$ & March to June; Aug. to Nov. \\
\hline \multirow[t]{4}{*}{ Convolvulaceae } & Ipomoea bahiensis Willd. & - & Ter & $\mathrm{Z}$ & $\mathrm{N}$ & $\mathrm{CA}$ & Jan. to Dec. \\
\hline & Ipomoea sp. & Beach Morning Glory/Goat's foot & $\mathrm{Li}$ & $\mathrm{Z}$ & $\mathrm{N}$ & $\mathrm{CC}, \mathrm{CA}, \mathrm{GM}$ & Jan. to Dec. \\
\hline & Merremia aegyptia (L.) Urb. & - & NR & NR & - & GM & Oct. \\
\hline & Merremia sp. & - & NR & NR & - & $\mathrm{CA}$ & Aug. \\
\hline Crassulaceae & Bryophyllum pinnatum (Lam.) Oken. & Horse chestnut & Herb & $\mathrm{Z}$ & - & $\mathrm{CA}$ & July to Dec. \\
\hline \multirow[t]{3}{*}{ Cucurbitaceae } & Cayaponia tayuya (Vell.) Cogn. & Tayuya & $\mathrm{Li}$ & A & $\mathrm{N}$ & $\mathrm{CA}$ & Aug. \\
\hline & Momordica charantia $\mathrm{L}$. & Balsam pear & $\mathrm{Li}$ & A & $\mathrm{N}$ & $\mathrm{CA} ; \mathrm{CT}$ & July to Dec. \\
\hline & Sechium edule Swartz. & Chayote & $\mathrm{Li}$ & A & $\mathrm{N}$ & $\mathrm{CA}$ & March to July \\
\hline \multirow[t]{7}{*}{ Euphorbiaceae } & Croton campestris St. Hill. & Bellyache bush & Shr & A & $\mathrm{N}$ & $\mathrm{CA} ; \mathrm{CT}$ & Jan. to Feb.; May \\
\hline & Croton moritibensis Baill. & Croton & Shr & A & $\mathrm{N}$ & $\mathrm{CA} ; \mathrm{CT}$ & Aug. to Dec. \\
\hline & Croton lobatus L. & Croton & Herb & A & $\mathrm{N}$ & $\mathrm{CA}$ & May to Sep. \\
\hline & Croton sp.1 & - & Herb & A & $\mathrm{N}$ & $\mathrm{CA}$ & May to Sep. \\
\hline & Croton sp. 2 & Croton & Herb & A & $\mathrm{N} / \mathrm{P}$ & $\mathrm{CA}$ & March to June \\
\hline & Croton sp. 3 & Croton & NR & A & - & CA & Oct to Jan. \\
\hline & $\begin{array}{l}\text { Euphorbia hyssopifolia L. } \\
\text { Euphorbia milii L. }\end{array}$ & $\begin{array}{l}\text { Garden spurge } \\
\text { Coroa-de-cristo }\end{array}$ & $\begin{array}{l}\text { Herb } \\
\text { Shr }\end{array}$ & $\begin{array}{l}\text { A } \\
\text { A }\end{array}$ & - & $\begin{array}{l}\text { CA } \\
\text { CA }\end{array}$ & $\begin{array}{l}\text { April to July } \\
\text { May to Sep. }\end{array}$ \\
\hline
\end{tabular}


PLANTS VISITED BY Apis mellifera L. (HYMENOPTERA: APIDAE) IN RECÔNCAVO BAIANO, STATE OF BAHIA, BRAZIL

Table 1. Continue...List of species of apicultural interest collected at flowering within $1.500 \mathrm{~m}$ of apiaries in Recôncavo Baiano: March/2009 to February/2010.

\begin{tabular}{|c|c|c|c|c|c|c|c|}
\hline Family & Scientific Name & Common Name & $\mathrm{HC}$ & FS & $\mathbf{R E}$ & Place of collection & Flowering Period \\
\hline \multirow[t]{3}{*}{ Euphorbiaceae } & Jatropha molissima (Pohl) Baill. & Black vomit nut & Shr & A & - & $\mathrm{CT}$ & March \\
\hline & Manihot esculenta Crantz. & Brazilian arrowroot & Shr & A & $\mathrm{N}$ & $\mathrm{CA}$ & Aug. \\
\hline & Ricinus communis $\mathrm{L}$. & Castor bean & Shr & $\mathrm{A}$ & $\mathrm{N} / \mathrm{P}$ & $\mathrm{CA}$ & Aug. to Dec. \\
\hline \multirow[t]{7}{*}{ Lamiaceae } & Hyptis multiflora Pohl. & Bishop's wort & Herb & $\mathrm{Z}$ & $\mathrm{N}$ & $\mathrm{CA}$ & May to July \\
\hline & Ocimum basilicum $\mathrm{L}$. & Common Basil & Herb & $\mathrm{Z}$ & $\mathrm{N}$ & $\mathrm{CA}$ & Aug. to Dec. \\
\hline & Ocimum canum Sims. & Sweet basil & Herb & $\mathrm{Z}$ & $\mathrm{N}$ & $\mathrm{CT}$ & May to June \\
\hline & Ocimum gratissimum $\mathrm{L}$. & Basil & Shr & $\mathrm{Z}$ & $\mathrm{N}$ & $\mathrm{CA}$ & Nov. \\
\hline & Ocimum sp. & - & Herb & $\mathrm{Z}$ & $\mathrm{N}$ & $\mathrm{CA}$ & March to April \\
\hline & Salvia sp.1 & - & Herb & $\mathrm{Z}$ & $\mathrm{N}$ & $\mathrm{CA}$ & July to Sep. \\
\hline & Salvia sp.2 & Salvia & Herb & $\mathrm{Z}$ & $\mathrm{N}$ & $\mathrm{CA}$ & May. to April \\
\hline Lauraceae & Persea americana Mill. & Avocado pear & Arb & A & - & $\mathrm{CA}$ & Sep \\
\hline \multirow[t]{12}{*}{ Leguminosae-Caesalpinioideae } & Bauhinia fortificta Link. & Brazilian orchid tree & Arb & $\mathrm{Z}$ & $\mathrm{P}$ & CA & March to June; Sep. to Feb. \\
\hline & Caesalpinia ferrea Mart. & Brazilian Iron wood & Arb & $\mathrm{Z}$ & $\mathrm{N}$ & IT & Jan. to March \\
\hline & Caesalpinia peltophoroides Benth. & Sibipiruna & Arb & $\mathrm{Z}$ & $\mathrm{P}$ & $\mathrm{CA}$ & Jan. to March \\
\hline & Caesalpinia pyramidalis Tul. & Lobelia wall & Arb & $\mathrm{Z}$ & $\mathrm{P}$ & $\mathrm{CT}$ & Jan. to April \\
\hline & Delonix regia Raff. & Flamboyant & Arb & $\mathrm{Z}$ & $\mathrm{P}$ & $\mathrm{CA}$ & Nov. to Jan. \\
\hline & $\begin{array}{l}\text { Poeppigia procera C. Presl. } \\
\text { Senna macranthera (Collad.) H.S.Irwin \& }\end{array}$ & - & Arb & $\mathrm{Z}$ & $\mathrm{N}$ & IT & $\begin{array}{c}\text { Aug. to Oct. } \\
\text { Jan. to Feb.; May; July to }\end{array}$ \\
\hline & Barneby & Java bean & Arb & $\mathrm{Z}$ & $\mathrm{P}$ & $\mathrm{CC}, \mathrm{CA}, \mathrm{CT}$ & Aug. \\
\hline & Senna obtusifolia $\mathrm{L}$. & Java bean & Shr & $\mathrm{Z}$ & $\mathrm{P}$ & $\mathrm{CC}, \mathrm{CA}, \mathrm{GM}$ & Aug. to Dec. \\
\hline & Senna occidentalis L. & Stinking weed & Shr & $\mathrm{Z}$ & $\mathrm{P}$ & $\mathrm{CC}, \mathrm{CA}, \mathrm{GM}$ & March to April \\
\hline & Senna spectabilis DC. & Bougainvillea Willd & Arb & $\mathrm{Z}$ & $\mathrm{P}$ & IT & Dec. to April \\
\hline & Senna sp. & Cassia flowers & Shr & $\mathrm{Z}$ & $\mathrm{P}$ & CA & March to May; Oct. to Dec. \\
\hline & Tamarindus indica $\mathrm{L}$. & Tamarindo & Arb & $\mathrm{Z}$ & $\mathrm{P}$ & $\mathrm{CC}, \mathrm{CA}, \mathrm{GM}$ & Nov. to Jan. \\
\hline \multirow[t]{6}{*}{ Leguminosae-Faboideae } & Bowdichia virgilioides Kunth. & Sucupira & Arb & $\mathrm{Z}$ & $\mathrm{P}$ & $\mathrm{CA} ; \mathrm{CT}$ & Nov. \\
\hline & Cajanus cajans $\mathrm{L}$. & Hybanthus & Herb & $\mathrm{Z}$ & $\mathrm{P}$ & $\mathrm{CA}$ & Sep. to Feb. \\
\hline & Calliandra surinamensis Benth. & - & Arb & $\mathrm{Z}$ & - & $\mathrm{CC}$ & Sep. \\
\hline & Canavalia ensiformis DC. & Jack bean & Herb & $\mathrm{Z}$ & - & $\mathrm{CA}$ & Sep. to Nov. \\
\hline & Canavalia sp. & Candida & Herb & $\mathrm{Z}$ & - & $\mathrm{CC}$ & Sep. \\
\hline & Chaetocalyx scandens L. & - & Ter & $\mathrm{Z}$ & $\mathrm{N}$ & IT & - \\
\hline
\end{tabular}


Table 1. Continue...List of species of apicultural interest collected at flowering within $1.500 \mathrm{~m}$ of apiaries in Recôncavo Baiano: March/2009 to February/2010.

\begin{tabular}{|c|c|c|c|c|c|c|c|}
\hline Family & Scientific Name & Common Name & $\mathrm{HC}$ & FS & $\mathbf{R E}$ & Place of collection & Flowering Period \\
\hline \multirow{21}{*}{ Leguminosae-Faboideae } & Chamaecrista belemii (H.S.Irwin \& Barneby ) & & & & & & \\
\hline & H.S.Irwin \& Barneby & - & Herb & $\mathrm{Z}$ & $\mathrm{P}$ & IT & - \\
\hline & Chamaecrista flexuosa (L.) Greene & - & Herb & $\mathrm{Z}$ & $\mathrm{P}$ & $\mathrm{CA}$ & May to July \\
\hline & Chamaecrista rotundifolia (Pers.) Greene & - & Herb & $\mathrm{Z}$ & $\mathrm{P}$ & $\mathrm{CA}$ & May to July \\
\hline & Chamaecrista sp. & - & Herb & $\mathrm{Z}$ & $\mathrm{P}$ & $\mathrm{CA}$ & Aug. to Dec. \\
\hline & Clitoria fairchildiana Howard. & Sombrero & Arb & $\mathrm{Z}$ & $\mathrm{P}$ & $\mathrm{CA}$ & Nov. \\
\hline & Crotalaria incana $\mathrm{L}$. & Rattlepod & Herb & $\mathrm{Z}$ & $\mathrm{P}$ & CA, GM & Feb. to May \\
\hline & Desmodium adscendens (Sw.) DC. & Desmodium & Herb & $\mathrm{Z}$ & $\mathrm{P}$ & $\mathrm{CC}, \mathrm{CA}, \mathrm{GM}$ & Aug. to Jan. \\
\hline & Desmodium sp. & Desmodium & Herb & $\mathrm{Z}$ & - & $\mathrm{CA}$ & March to Nov. \\
\hline & Desmodium triflorum (L.) DC. & Desmodium & Herb & $\mathrm{Z}$ & $\mathrm{P}$ & $\mathrm{CT}$ & Jan.; April; July \\
\hline & Erythrina velutina Willd. & Woodson & Arb & $\mathrm{Z}$ & $\mathrm{P}$ & $\mathrm{CT}$ & Nov. to Dec. \\
\hline & Gliricidia sepium Jacq. & Quick-stick & Arb & $\mathrm{Z}$ & $\mathrm{N}$ & $\mathrm{CA}$ & Aug. to Sep. \\
\hline & Indigofera hirsuta $\mathrm{L}$. & Hairy indigo & Herb & $\mathrm{Z}$ & $\mathrm{P}$ & $\mathrm{CA}$ & May to July \\
\hline & $\begin{array}{l}\text { Lablab purpureus (L.) Sweet. } \\
\text { Lonchocarpus cultratus (Vell.) A.M.G. Azevedo }\end{array}$ & Hyacinth bean & Herb & $\mathrm{Z}$ & $\mathrm{P}$ & CA & Aug. \\
\hline & Macroptilium sp.1 & Willow & $\mathrm{Li}$ & $\mathrm{Z}$ & $\mathrm{P}$ & $\mathrm{CA}$ & Aug. to Nov. \\
\hline & Macroptilium sp.2 & - & Herb & $\mathrm{Z}$ & $\mathrm{P}$ & $\mathrm{CA}$ & May to July \\
\hline & Peltophorum dubium (Spreng) Taub. & Snake cactus & Arb & $\mathrm{Z}$ & $\mathrm{P}$ & $\mathrm{CA}$ & Nov. \\
\hline & Phaseolus vulgaris L. & Phellodendron & Herb & $\mathrm{Z}$ & - & $\mathrm{CA}$ & April to May; Sep. to Oct. \\
\hline & Stylosanthes viscosa $\mathrm{Sw}$. & Poor man's friend & Herb & $\mathrm{Z}$ & $\mathrm{P}$ & $\mathrm{CA}$ & Aug. \\
\hline & Vigna unguiculata (L.) Walp. & Cylindrica & Herb & $\mathrm{Z}$ & $\mathrm{P}$ & $\mathrm{CA}$ & July \\
\hline & Zornia diphylla Pers. & - & Herb & $\mathrm{Z}$ & $\mathrm{P}$ & $\mathrm{CA}$ & May to Sep. \\
\hline \multirow[t]{8}{*}{ Leguminosae-Mimosoideae } & Acacia bahiensis Benth. & Cat's claw & Shr & A & - & $\mathrm{CT}$ & Jan. to Feb. \\
\hline & Acacia langsdorfii Benth. & Cat's claw & Shr & A & - & $\mathrm{CT}$ & Jan.; Sep. to Oct. \\
\hline & Acacia sp. & Calumbi & Arb & A & $\mathrm{N} / \mathrm{P}$ & $\mathrm{CA}$ & Feb. \\
\hline & Albizia polycephala (Benth.) Killip & Angico-branco & Arb & A & - & $\mathrm{CT}$ & Dec. \\
\hline & AnadenantHerba colubrina (Vell.) Brenan & Angico & Arb & A & - & $\mathrm{CA} ; \mathrm{CT}$ & Jan. \\
\hline & Inga bahiensis Benth. & Ingá & Arb & A & - & $\mathrm{CA} ; \mathrm{CT}$ & Nov. \\
\hline & Inga edulis Mart. & Ingá-cipó & Arb & A & - & $\mathrm{CA}$ & Aug. to Dec. \\
\hline & Leucaena leucocephala Lam. & Leucena & Arb & A & $\mathrm{N} / \mathrm{P}$ & $\mathrm{CA}$ & Sep. to Dec. \\
\hline
\end{tabular}


PLANTS VISITED BY Apis mellifera L. (HYMENOPTERA: APIDAE) IN RECÔNCAVO BAIANO, STATE OF BAHIA, BRAZIL

Table 1. Continue...List of species of apicultural interest collected at flowering within $1.500 \mathrm{~m}$ of apiaries in Recôncavo Baiano: March/2009 to February/2010.

\begin{tabular}{|c|c|c|c|c|c|c|c|}
\hline Family & Scientific Name & Common Name & $\mathrm{HC}$ & FS & $\mathbf{R E}$ & Place of Collection & Flowering Period \\
\hline \multirow[t]{3}{*}{ Leguminosae-Mimosoideae } & Mimosa caesalpiniaefolia Benth. & Thrus & Arb & $\mathrm{A}$ & - & $\mathrm{CC}, \mathrm{CA}$ & June to Sep. \\
\hline & Mimosa pudica $\mathrm{L}$. & Malícia & Herb & A & $\mathrm{N} / \mathrm{P}$ & $\mathrm{CC}, \mathrm{CA}$ & April to July and Aug. to Nov. \\
\hline & Prosopis juliflora DC. & Mesquite & Arb & A & - & $\mathrm{CA} ; \mathrm{CT}$ & Oct. to Dec. \\
\hline Liliaceae & Bulbine frutescens $\mathrm{L}$. & Snake flower & Herb & A & - & $\mathrm{CA}$ & May to Aug. \\
\hline Loranthaceae & Struthanthus sp. & Mistletoe & $\mathrm{Li}$ & A & - & $\mathrm{CA}$ & Sep. to Nov. \\
\hline Lythraceae & Cuphea racemosa (L.f.) Spreng. & - & Herb & $\mathrm{Z}$ & - & $\mathrm{CT}$ & April to June \\
\hline \multirow[t]{2}{*}{ Malpighiaceae } & Byrsonima sp. & Golden spoon & Shr & $\mathrm{Z}$ & - & CA & Dec. \\
\hline & Stigmaphyllon auriculatum (Cav.) Adr. Juss. & - & Ter & $\mathrm{Z}$ & $\mathrm{P}$ & IT & Dec. to Feb. \\
\hline \multirow[t]{16}{*}{ Malvaceae } & Gossypium hirsutum $\mathrm{L}$. & Cotton & Herb & A & $\mathrm{P}$ & $\mathrm{CA}$ & Oct. to Jan. \\
\hline & Herissantia crispa (L.) Fryxell & Bladdermallow & Herb & A & - & $\mathrm{CT}$ & March; June; Aug. to Oct. \\
\hline & Hibiscus esculentus L. & Ókra & Herb & A & $\mathrm{P}$ & $\mathrm{CA}$ & Dec. to Feb. \\
\hline & Hibiscus rosa-sinensis $\mathrm{L}$. & Graxa & Shr & A & $\mathrm{N} / \mathrm{P}$ & $\mathrm{CA}, \mathrm{CC}$ & Jan. to Dec. \\
\hline & Hibiscus sp. & Hibiscus & Shr & A & $\mathrm{P}$ & $\mathrm{CA}$ & April to Aug. \\
\hline & Malvastrum tomentosum (L.) S.R. Hill & - & Herb & A & - & $\mathrm{CT}$ & Aug. \\
\hline & Sida cordifolia $\mathrm{L}$. & Arrow leaf sida & Herb & A & $\mathrm{P}$ & $\mathrm{CA}$ & Jan. to Dec. \\
\hline & Sida linifolia Juss ex Card. & Arrow leaf sida & Herb & A & $\mathrm{P}$ & $\mathrm{CA}$ & Aug. to Dec. \\
\hline & Sida paniculata $\mathrm{L}$. & Arrow leaf sida & Herb & A & $\mathrm{P}$ & CT & Jan.; Aug. to Sep. \\
\hline & Sida rhombifolia $\mathrm{L}$. & Arrow leaf sida & Herb & A & $\mathrm{P}$ & $\mathrm{CA}$ & March to June \\
\hline & Sida spinosa $\mathrm{L}$. & Ivy-leaf Sida & Herb & A & $\mathrm{P}$ & $\mathrm{CA}$ & July to Aug.; Oct. to Nov. \\
\hline & Sida sp.1 & Ivy-leaf Sida & Herb & A & $\mathrm{P}$ & $\mathrm{CA}$ & Aug. to Dec. \\
\hline & Sida sp.2 & Ivy-leaf Sida & Herb & A & $\mathrm{P}$ & CA & Aug. to Dec. \\
\hline & Sida sp.3 & Malv Ivy-leaf Sida & Herb & A & $\mathrm{P}$ & $\mathrm{CA}$ & March to May and Aug. to Nov. \\
\hline & Sida sp.4 & Ivy-leaf Sida & Herb & A & - & CA & Jan. to Dec. \\
\hline & Urena lobata $\mathrm{L}$. & Urens & Herb & A & - & CA & April to May; Aug.; Nov. \\
\hline Meliaceae & Trichilia hirta L. & Erytroxilum & Arb & A & - & CT & Jan. \\
\hline Molluginaceae & Mollugo verticillata $\mathrm{L}$. & - & Herb & A & - & $\mathrm{CA}$ & May \\
\hline Moringaceae & Moringa oleifera Lam. & Horseradish tree & Shr & A & $\mathrm{N} / \mathrm{P}$ & $\mathrm{CA}$ & Jan. a dez. \\
\hline Myrtaceae & $\begin{array}{l}\text { Eucalyptus sp. } 1 \\
\text { Eucalyptus sp. } 2\end{array}$ & $\begin{array}{l}\text { Eucalyptus } \\
\text { Eucalyptus }\end{array}$ & $\begin{array}{l}\text { Arb } \\
\text { Arb }\end{array}$ & $\begin{array}{c}\mathrm{A} \\
\mathrm{A}\end{array}$ & $\begin{array}{l}\mathrm{N} / \mathrm{P} \\
\mathrm{N} / \mathrm{P}\end{array}$ & $\begin{array}{l}\text { CA } \\
\text { CA }\end{array}$ & $\begin{array}{l}\text { Nov. to Dec.; Jan. to March } \\
\text { Nov. to Dec.; Jan.to March }\end{array}$ \\
\hline
\end{tabular}


Table 1. Continue...List of species of apicultural interest collected at flowering within 1.500 m of apiaries in Recôncavo Baiano: March/2009 to February/2010.

\begin{tabular}{|c|c|c|c|c|c|c|c|}
\hline Family & Scientific Name & Common Name & HC & FS & $\mathbf{R E}$ & Place of collection & Flowering Period \\
\hline \multirow[t]{6}{*}{ Myrtaceae } & Eucalyptus sp. 3 & Eucalyptus & Arb & A & $\mathrm{N} / \mathrm{P}$ & $\mathrm{CA}$ & Nov. to Dec.; Jan. to March \\
\hline & Eucalyptus torelliana F.Muell. & Eucalyptus & Arb & A & $\mathrm{N} / \mathrm{P}$ & $\mathrm{CA}$ & Nov. to Dec.; Jan. toMarch \\
\hline & Myrciaria cauliflora Berg. & Brazilian grape tree & Shr & A & - & $\mathrm{CA}$ & Sep. to Nov. \\
\hline & Psidium araça Raddi. & Arnica & Shr & A & $\mathrm{N} / \mathrm{P}$ & $\mathrm{CA}$ & March to April \\
\hline & Psidium guajava $\mathrm{L}$. & Guave & Arb & A & - & $\mathrm{CA}$ & Sep. \\
\hline & Syzygium malaccensis $\mathrm{L}$. & Malay apple & Arb & A & $\mathrm{N} / \mathrm{P}$ & $\mathrm{CA}$ & Nov. to Dec. \\
\hline Onagraceae & Ludwigia sp. & - & Herb & A & $\mathrm{N}$ & $\mathrm{CA}$ & April to May; Dec. to Jan. \\
\hline Oxalidaceae & Averrhoa carambola $\mathrm{L}$. & Star fruit & Arb & A & - & $\mathrm{CA}$ & Nov. \\
\hline Oxalidaceae & Oxalis psoraleoides H.B.K. & - & Shr & A & - & $\mathrm{CT}$ & Jan. to Feb.; May \\
\hline Papaveraceae & Argemone mexicana $\mathrm{L}$. & Prickly poppy & Herb & A & - & $\mathrm{CA}$ & Aug. \\
\hline \multirow[t]{3}{*}{ Passifloraceae } & Passiflora edulis L. & Passion fruit & Ter & A & - & $\mathrm{CA}$ & Oct. \\
\hline & Passiflora foetida $\mathrm{L}$. & Passion fruit & Ter & A & $\mathrm{N}$ & IT & Oct. \\
\hline & Passiflora violacea Vell. & Passion fruit vine & Ter & A & $\mathrm{N}$ & IT & Oct. \\
\hline \multirow[t]{2}{*}{ Poaceae } & Sorghum bicolor $\mathrm{L}$. & Great millet & Herb & NR & $\mathrm{P}$ & $\mathrm{CA}$ & Oct. \\
\hline & Zea mays L. & Corn & Herb & NR & $\mathrm{P}$ & $\mathrm{CC}, \mathrm{CA}, \mathrm{GM}$ & Aug. \\
\hline \multirow[t]{3}{*}{ Polygonaceae } & Antigonon leptopus Hook. & Coral creeper & Ter & A & - & $\mathrm{CA}$ & Jan. to Dec. \\
\hline & Coccoloba paniculata Meisn. & Arborecens & Shr & A & - & $\mathrm{CC}$ & Sep. \\
\hline & Ruprechtia laxiflora Meisn. & - & Arb & A & - & $\mathrm{CT}$ & Dec.; Jan. to Feb. \\
\hline \multirow[t]{4}{*}{ Portulacaceae } & Portulaca elatior Mart. & - & Herb & A & - & $\mathrm{CT}$ & Jan. to June.; Aug. to Oct. \\
\hline & Portulaca oleracea L. & Garden purslane & Herb & A & $\mathrm{P}$ & $\mathrm{CC}, \mathrm{CA}, \mathrm{GM}$ & Sep to Nov. and March to June \\
\hline & Portulaca sp. & - & Herb & A & $\mathrm{N}$ & $\mathrm{CA}$ & Marc to June \\
\hline & Talinum patens Will. & Gaertner & Herb & A & - & $\mathrm{CC}, \mathrm{CA} ; \mathrm{CT}$ & March to May and Aug. to Nov \\
\hline Rhamnaceae & Ziziphus joazeiro Mart. & Ziziphus jujuba Miller & Arb & A & $\mathrm{N}$ & $\mathrm{CT}$ & Dec.; Jan.to Feb. \\
\hline \multirow[t]{5}{*}{ Rubiaceae } & Borreria suaveolens G. Mey & Suaveolens Var & Herb & A & $\mathrm{N}$ & $\mathrm{CA}$ & May to Oct. \\
\hline & Borreria verticillata (L.) G. Mey. & Ambrosioides & Herb & A & $\mathrm{N} / \mathrm{P}$ & $\mathrm{CA}, \mathrm{CC}, \mathrm{SAF}, \mathrm{GM}$ & May to Aug. \\
\hline & Borreria sp. & - & Herb & A & $\mathrm{N} / \mathrm{P}$ & $\mathrm{CA}$ & May \\
\hline & Emmeorhiza umbellata (Spreng.) K. Schum. & - & $\mathrm{Li}$ & A & - & $\mathrm{CC}$ & Sep. \\
\hline & Genipa americana $\mathrm{L}$. & Genipa tree & Arb & $\mathrm{A}$ & $\mathrm{N}$ & $\mathrm{CA}$ & March to May \\
\hline
\end{tabular}


PLANTS VISITED BY Apis mellifera L. (HYMENOPTERA: APIDAE) IN RECÔNCAVO BAIANO, STATE OF BAHIA, BRAZIL

Table 1. Continue...List of species of apicultural interest collected at flowering within 1.500 m of apiaries in Recôncavo Baiano: March/2009 to February/2010.

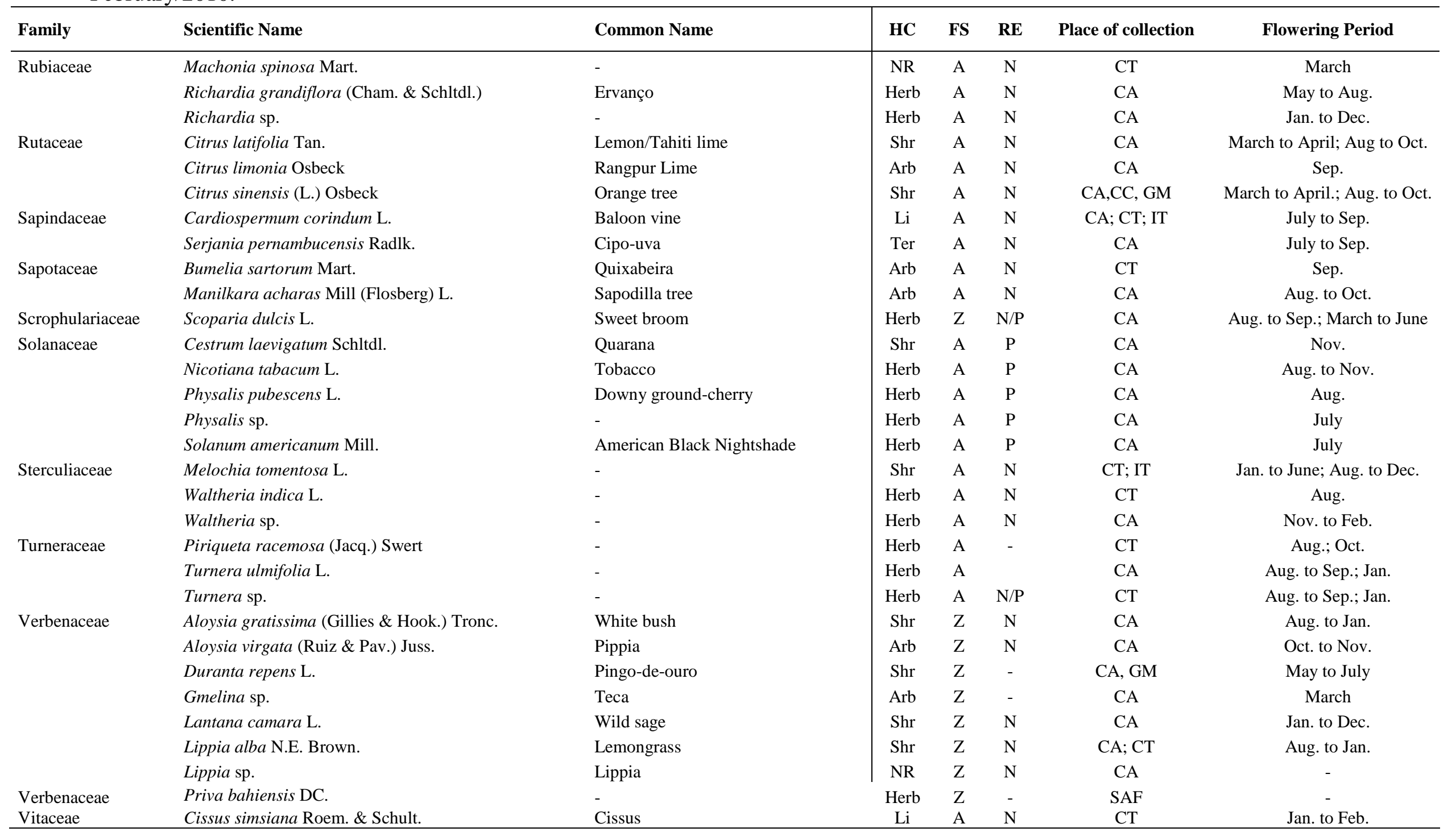




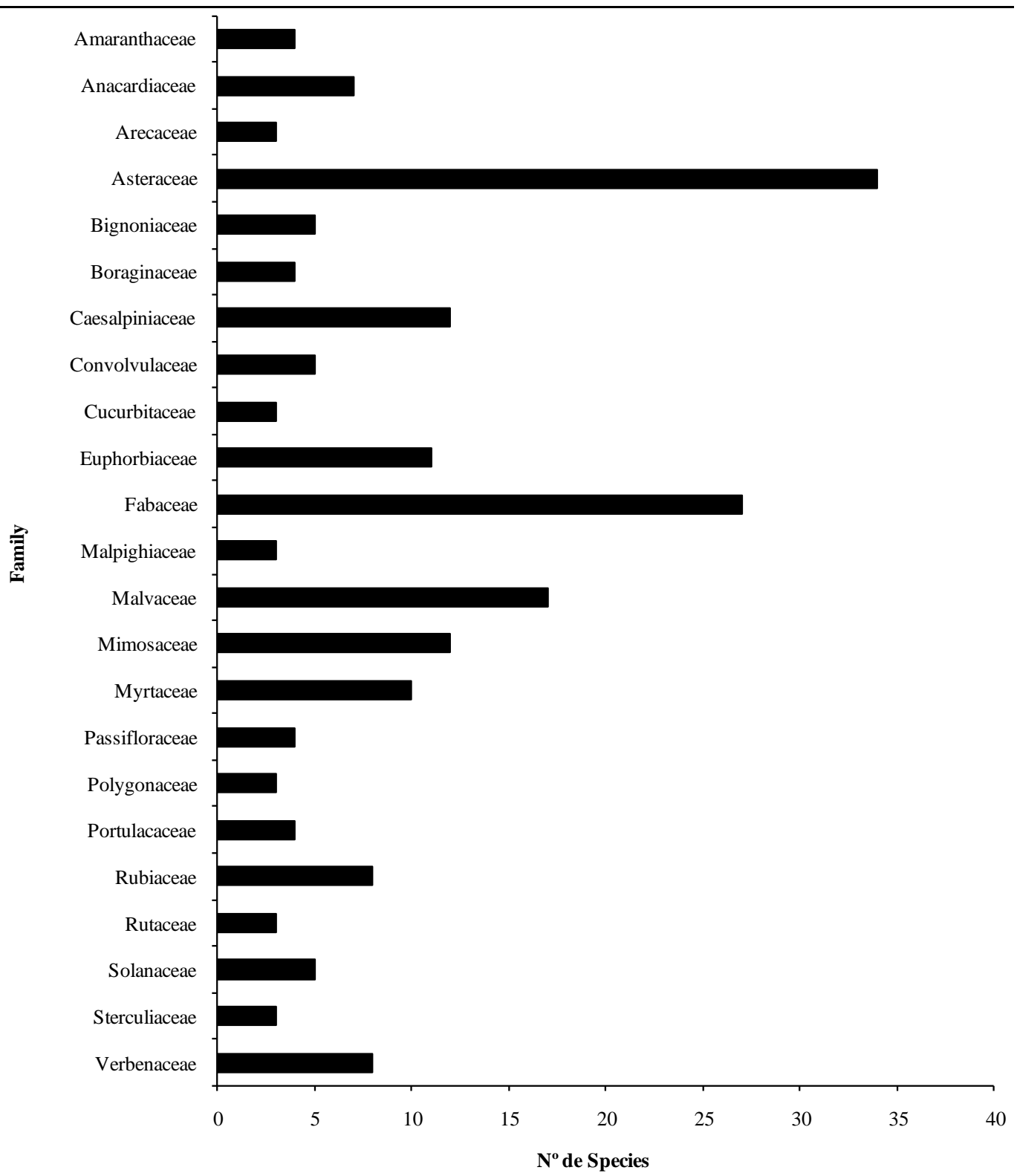

Figure 1. Main families in number of species of apicultural interest collected at flowering plants within $1.500 \mathrm{~m}$ of apiaries in Recôncavo Baiano: March/2009 to February/2010.

Aguiar et al. (1995) when evaluating the floral resources used by bees in the caatinga area found that the families Boraginaceae, Convolvulaceae, Cactaceae, Sterculiaceae and Caesalpiniaceae represent the largest source of pollen and nectar in the area. According to these authors the floral resources are related to seasonal variation and the flowering pattern of plants exerts a strong influence on the community structure of bees.

For the Myrtaceae family, pollen is the main resource offered to the pollinators, being the primary resource through which the bees, probably the most important group of pollinators of this family, visit the flowers (NIC LUGHADHA \& PROENÇA, 1996). 
There are very few evidence of nectar production observed in Myrciaria dubia (PETERS \& VASQUEZ (1986, 1987), MAUÉS \& COUTURIER (2002), Psidium guajava and Eugenia spp. (RAMALHO et al., 1990), Myrciaria cauliflora (MALERBO et al., 1991) and Plinia glomerata (PIRANI \& CORTOPASSI-LAURINO, 1993). This family was represented by 10 species in this study with emphasis on the Eucalyptus genus with 4 species (Table 1 and Figure 1). Some Eucalyptus species are cited by NougueiraNeto $(1953,2009)$ as species producing good quality honey.

Among the plants of the family Verbenaceae, Aloysia virgata is considered by Brandão et al. (2002) as a species of great importance to apiculture. It is found in Bahia, Mato Grosso do Sul and in the Southeast (LORENZ, 2002). This species, which was collected in the city of Cruz das Almas, Bahia, can be used by beekeepers in the area around the apiary to produce honey.

The genera with the highest diversity were Sida with 9 species, Croton (6), Senna (5) and Chamaecrista, Eucalyptus, Eupatorium, Ocimum, Passiflora and Vernonia with four species each (Table 1).

From the species collected in this study, 64 were already registered as constituents of apicultural flora in different localities of Bahia 110 in studies conducted by Moreti et al. (2000); Sodre et al. (2001); Costa (2002); Almeida et al. (2006); Melo (2008); Novais et al. (2009) and Oliveira et al. (2010).

The highest percentage of plants that were identified had an herbaceous growth habit (44\%), followed by a woody habit (26\%) and shrubs (18\%). Lianas (4\%), vines (4\%) and palms (1\%) were significantly less represented. Most of the bees visiting the flowers of herbaceous plants can be associated to the abundance of these plants in the region, the flowering period with a higher concentration of species between March and June during the rainy season in the region (Table 1), the shape and color of flowers (more attractive to bees) and even the concentration of nectar and pollen quantity offered by them. According to Oliveira et al. (2009) bees show a preference for woody plants in general and the resources to be used by the bees depend on their availability in the collection area. Rain promotes diversification of resources due to the low flowering.

Alves (2008), working with the characterization of apicultural flora of the upper Rio Paraná, found that the growth habit of the species was varied and reasonably well distributed, and the tree species and shrubs both represented $19.70 \%$ of the plants collected and the other subshrubs 26.52\%, $12.88 \%$ 
herbaceous and $21.21 \%$ vines; results somewhat no similar to those found in this study.

It was found that $65 \%$ of species had actinomorphic flowers and 33\% zygomorphic flowers, while $2 \%$ of the species did not have their floral symmetry determined during the period to data collecting. Alves (2008) reported in his study that the most common pattern in relation to floral symmetry was actinomorphic flowers arranged in inflorescences and with a light color corollas. Apis mellifera prefer actinomorphic flowers (DAFNI, 1992).

Some fruit species $(8 \%)$ of apicultural interest to the region were observed, such as Anacardium occidentale L., Byrsonima sp., Citrus latifolia Tan., C. limonia Osbeck, $C$. sinensis (L.) Osbeck, Eugenia uniflora L., Malphigia emarginata, Mangifera indica L., Manilkara acharas Mill (Flosberg) L., Myrciaria cauliflora Berg., Passiflora edulis, P. foetida L., P. macrocarpa Mart., Persea americana Mill., Psidium araca Raddi., $P$. guajava L., Spondias tuberosa Arr. Cam., Spondias sp., Syzygium cumini (L.) Skeels and Tamarindus indica L. (Table 1). In addition to using cultivated native fruit plants, many bees also visit flowers of ornamental plants, like Thunbergia grandiflora Roxb., Asystasia gangetica (L.) T. Anderson and Tecoma stans
(L.) Kunth (MILET-PINHEIRO \& SCHLINDWEIN, 2008).

According to Santos et al. (2006)

among the species preferentially visited by Apis mellifera in Petrolina, State of Pernambuco, the herbaceous species, such as Borreria verticillata (L.) G. Mey., Diodia teres Walter., Waltheria rotundifolia Schrank, Merremia aegyptia L., Jacquemontia confusa Meisn., and Hypenia salzmanni Benth stood out as excellent suppliers of nectar during the rainy season. Some of these species appear in the list of plants collected in this study and the species Borreria verticillata is among the pollen types identified in honeys and the mass of pollen collected by Africanized honeybees in Bahia (COSTA, 2002; NASCIMENTO et al., 2009b).

Carvalho \& Marchini (1999), in a study on plants visited by Apis mellifera in Castro Alves, Bahia, also found the occurrence of several herbaceous species such as Commelina benghalensis L., Croton campestris St. Hil., Centratherum punctatum Cass., Momordica charantia L., Sida paniculata L., Portulaca spp. and Waltheria indica L., and although they bloom during the rainy season and despite being considered weeds to crops, they present an apicultural potential. These species were also collected in the region studied and the 
record of flowering ones (Table 1) corroborates with those of the authors.

\section{CONCLUSION}

Flora visited by Apis mellifera L. in the Recôncavo Baiano region has a high diversity of species, especially from the family Leguminosae.

\section{ACKNOWLEDGEMENTS}

The authors would like to thank the National Council for Scientific and Technological Development (CNPq), Coordination for the Improvement of Higher Education Personnel (CAPES) and Foundation for Research Support of the State of Bahia (FAPESB) for their financial support and scholarships.

\section{REFERENCES}

AGUIAR, C. M. L. 2003. Utilização de recursos florais por abelhas (Hymenoptera, Apoidea) em uma área de Caatinga (Itatim, Bahia, Brasil). Revista Brasileira de Zoologia, Curitiba, v.20, n. 3, p.457-467.

AGUIAR, C. M. L.; MARTINS, C. F.; MOURA, A. A. 1995. Recursos florais utilizados por abelhas (Hymenoptera, Apoidea) em áreas de caatinga (São João do Cariri, Paraíba). Revista Nordestina de
Biologia, João Pessoa, v.10, n. 2, p.101117.

ALMEIDA, D. 2002. Espécies de abelhas (Hymenoptera, Apoidea) e tipificação dos méis por elas produzidos em área de cerrado no município de Pirassununga, Estado de São Paulo. 103 p. Dissertação (Mestrado em Entomologia) - Escola Superior de Agricultura Luiz de Queiroz, Piracicaba.

ALMEIDA, S. M. M.; CARVALHO, C. A. L.; ABREU, R. D.; SANTOS, F. A. R.; SILVA, A. M.; OLIVEIRA, P. P.; ARAÚJO, R. C. M. S. 2006. Plantas visitadas por Apis mellifera L. em NovaSoure, Bahia. Magistra, Cruz das Almas, v.18, n.3, p.152-161.

ALVES, E. M. 2008. Identificação da flora e caracterização do mel orgânico de abelhas africanizadas das ilhas Floresta e Laranjeira, do alto rio Paraná. 77 p. Tese (Doutorado em Zootecnia) Universidade Estadual de Maringá.

ANDENA, S. R.; BEGO, L. R.; MECHI, M. R. 2005. A comunidade de abelhas (Hymenoptera: Apoidea) de uma área de cerrado (Corumbataí-SP) e suas visitas às flores. Revista Brasileira de Zoociência, Juiz de Fora, V.7, n.1, p.55-91.

BELL, A. D. 1991. Plant Form: an illustrated guide to flowering plant 
morphology. New York: Oxford, University Press, 341p.

BRANDÃO, M.; LACA-BUENDIA, J. P.; MACEDO, J. F. 2002. Árvores Nativas e Exóticas do Estado de Minas Gerais. EPAMIG, Belo Horizonte-MG. 527p.

CARVAlHO, C. A. L.; MORETI, A. C. C. C.; MARCHINI, L. C.; ALVES, R. M. O.; OLIVEIRA, P. C. F. 2001. Pollen spectrum of samples of uruçu bee (Melipona scutellaris Latreille, 1811) honey. Revista Brasileira de Biologia, São Carlos, v.61, n. 3, p.63-67.

CARVALHO, C. A. L.; MARCHINI, L. C. 1999. Plantas visitadas por Apis mellifera L. no vale do rio Paraguaçu, Município de Castro Alves, Bahia. Revista Brasileira de Botânica, São Paulo, v.22, n. 2, p.333-338. CARVAlHO, C. A. L.; NASCIMENTO, A. S.; PEREIRA, L. L.; MACHADO, C. S.; CLARTON, L. 2006. Fontes nectaríferas e poliníferas utilizadas por Melipona quadrifasciata (Hymenoptera: Apidae) no Recôncavo Baiano. Magistra, Cruz das Almas, v.18, n. 3, p.249-256.

COSTA, J. B. A. 2002. Fontes de pólen utilizadas por operárias de Apis mellifera L. no município de Cruz das Almas-BA. 65 p. Dissertação (Mestrado em Ciências Agrárias) - Universidade Federal da Bahia, Cruz das Almas.
DAFNI, A. 1992. Pollination Ecology: a practical approach. Pergamon Press, Oxford. 243p.

ERDTMAN, G. 1960. The acetolysis method. A revised description. Svensk Botanisk Tidskrift, Stockholm v.39, p.561-564.

FERRI, M. G. 1983. Botânica: morfologia externa das plantas. São Paulo: Melhoramento, $4^{\mathrm{a}}$ ed. 149p.

JONES, G. D.; BRYANT JR, V. M. 1996. Melissopalynology. In: JANSONIUS, J. D. C.; MCGREGOR, P. C. (Ed.), Salt Lake City, Palynology: principles and applications. Salt Lake City: American Association of Stratigraphic Palynologists Foundation, p.933-938.

LOCATELLI, E.; MACHADO, I. C. 2001. Bee diversity and their floral resources in a fragment of a tropical altitudinal wet forest ("Brejos de altitude") in Northeastern Brazil. Acta Horticulturae, Hague, v.561, n.2, p.317-325.

LORENZI, H. 2002. Árvores Brasileiras Manual de Identificação e Cultivo de Plantas Arbóreas Nativas do Brasil, Nova Odessa: Instituto Plantarum, $4^{\mathrm{a}}$ ed. 299p.

MALERBO, D. T. S.; TOLEDO, V. A. A.; COUTO, R. H. N. 1991. Polinização entomófila em jabuticabeira (Myrciaria cauliflora Berg.). Ciência Zootécnica, Jaboticabal, v.6, n. 1, p.3-5. 
MARCHINI, L. C.; SODRÉ, G. S.; MORETI, A. C. C. C. 2004. Mel brasileiro: Composição e normas. Ribeirão Preto: ASP, 131p.

MARCHINI, L. C.; MORETI, A. C. C. C.; TEIXEIRA, E. W.; SILVA, E. C. A.; RODRIGUES, R. R.; SOUZA, V. C. 2001. Plantas visitadas por abelhas africanizadas em duas localidades do estado de São Paulo. Scientia Agrícola, Piracicaba, v.58, n. 3, p.413-420.

MAUÉS, M. M.; COUTURIER, G. 2002. Biologia floral e fenologia reprodutiva do camu-camu (Myrciaria dubia (H.B.K.) McVaugh, Myrtaceae) no Estado do Pará, Brasil. Revista Brasileira de Botânica, São Paulo, v.25, n. 4, p.441-448.

MELO, P. A. 2008. Flora apícola em Jequitibá, Mundo Novo-BA. Dissertação (Mestrado em Ciências Agrárias) Universidade Federal do Recôncavo da Bahia, Cruz das Almas. 87 p.

MILET-PINHEIRO, P.; SCHLINDWEIN, C. 2008. Comunidade de abelhas (Hymenoptera, Apoidea) e plantas em uma área do Agreste pernambucano, Brasil. Revista Brasileira de Entomologia, São Paulo, v.52, n. 4, p.625-636.

MORETI, A. C. C. C.; MARCHINI, L. C.; TEIXEIRA, E. W. Caracterização de plantas apícolas do centro de Apicultura
Tropical/Instituto de Zootecnia, em Pindamonhangaba, SP. In: CONGRESSO BRASILEIRO DE APICULTURA, 12., Salvador, 1998. Anais..., Salvador: Confederação Baiana de Apicultura, 1998. p.179.

MORETI, A. C. C. C.; CARVALHO, C. A. L.; OLIVEIRA, P. C. F.; MARCHINI, C. L. 2000. Botânica e fisiologia vegetal. Espectro polínico de amostras de méis de Apis mellifera L. coletadas na Bahia. Bragantia, Campinas, v.59, n. 3, p.1-6.

MUNIZ, F. H.; BRITO, E. R. 2007. Levantamento da flora apícola do município de Itapecuru-Mirim, Maranhão. Revista Brasileira de Biociências, Porto Alegre, v.5, n. 1, p.111-113.

NASCIMENTO, A.S.; CARVALHO, C. A. L.; SODRÉ, G. S.; PEREIRA, L. L.; MACHADO, C. S.; JESUS, L. S. 2009a. Recursos nectaríferos e poliníferos explorados por Melipona quadrifasciata anthidioides em Cruz das Almas, Bahia. Magistra, Cruz das Almas, v.21, n. 1, p.25-29.

NASCIMENTO, A. S.; SANTOS, P. C.; SODRÉ, G. S.; CARVALHO, C. A. L.; CLARTON, L. 2009b. Espectro polínico de amostras de méis de Apis mellifera L. do município de Biritinga, região semi-árida, Estado da Bahia. In: CONGRESSO 
NORDESTINO DE APICULTURA E MELIPONICULTURA, 1., 2009, Salvador. Anais..., Salvador: CBA, p.63. NIC LUGHADHA, E. N.; PROENÇA, C. 1996. A survey of the reproductive biology of the Myrtoideae (Myrtaceae). Annals of the Missouri Botanical Garden, Washington, v.83, n. 4, p.480-503.

NOGUEIRA-NETO, P. 1998. As abelhas e o meio ambiente In: Congresso Brasileiro de Apicultura, 12., 1998, Salvador. Anais..., Salvador, [S.1.]: Confederação Brasileira de Apicultura, p.49-155.

NOVAIS, J. S.; LIMA, L. C. L.; SANTOS, F. A. R. 2009. Botanical affinity of pollen harvested by Apis mellifera L. in a semiarid area from Bahia, Brazil. Grana, Stockholm, v.48, n. 3, p.224-234.

OLIVEIRA, F. P. M.; ABSY, M. L.; MIRANDA, I. S. 2009. Recurso polínico coletado por abelhas sem ferrão (Apidae, Meliponinae) em um fragmento de floresta na região de Manaus - Amazonas. Acta Amazonica, Manaus, v.39, n. 3, p.505518.

OLIVEIRA, P. P.; VAN DEN BERG, C.; SANTOS, F. A. R. 2010. Pollen analysis of honeys from Caatinga vegetation of the state of Bahia, Brazil. Grana, Stockholm, v.49, n. 1, p.67-75.
PETERS, C. M.; VASQUEZ, A. 1987. Estudios ecológicos de camu-camu (Myrciaria dubia). I. Producción de frutos en poblaciones naturales. Acta Amazônica, Manaus, v.16, n. 2, p.161174.

PIRANI, J. R.; CORTOPASSI-LAURINO, M. 1993. Flores e abelhas em São Paulo. São Paulo: Edusp/Fapesp, 194p.

POTT, A.; POTT, V. J. 1986. Inventário da flora apícola do Pantanal em Mato Grosso do Sul. Embrapa (ISSN 01027727), Corumbá.

RAMALHO, M.; KLEINERT-GIOVANNINI, A.; IMPERATRIZ-FONSECA, V. L. 1990. Important bee plants for stingless bees (Melipona and Trigonini) and Africanized honeybees (Apis mellifera) in neotropical habitats: a review. Apidologie, Paris, v.21, n.5, p.469-488.

SANTOS, R. F.; KIILL, L. H. P.; ARAÚJO, J. L. P. 2006. Levantamento da flora melífera de interesse apícola no município de Petrolina-PE. Revista Caatinga, Mossoró, v.19, n. 3, p.221-227.

SODRÉ, G. S.; MARCHINI, L. C.; MORETI, A. C. C. C.; CARVAlHO, C. A. L. 2001. Análises polínicas de méis de Apis mellifera L. 1758 (Hymenoptera: Apidae) do Litoral Norte do Estado da Bahia. 
Revista de Agricultura, Piracicaba, v.76, n. 2, p.215-225.

TORQUATO, T. G. M. 2006. Potencial da vegetação melitófila e abelhas associadas da área Olho D’água dos Pires, Esperantina, Piauí, Brasil. 61 p. Dissertação de Mestrado em Desenvolvimento e Meio Ambiente. Universidade Federal do Piauí, Teresina. VIANA, B. F.; SILVA, F. O. 2006. Polinização por abelhas em agroecossistemas, In: CONGRESSO BRASILEIRO DE
APICULTURA, 16., Aracaju. Anais..., Aracaju. 2006. CD-Rom.

VIANA, B. F.; SILVA, F. O.; KLEINERT, A. M. P. 2006. A flora apícola de uma área restrita de dunas litorâneas, Abaeté, Salvador, Bahia. Revista Brasileira de Botânica, São Paulo, v.29, n. 1, p.13-25.

WIESE, H. 1987. Nova Apicultura. Porto Alegre - RS: Agropecuária, 20ª ed., 428 p.

Recebido em: 25/01/2014 Aceito para publicação em: 12/08/2014 